\title{
Ex-ante Evaluation \\ von Investitionsalternativen
}

\author{
Am Beispiel von Wissenstransfer-, Lern- und Innovationsprozessen
}

\author{
Matthias Müller, Universität Hohenheim, Lehrstuhl für Innovationsökonomik, Wollgrasweg 23, \\ 70599 Stuttgart (m_mueller@uni-hohenheim.de) \\ Muhamed Kudic, Universität Bremen, Lehrstuhl für Volkswirtschaftslehre, insbes. Innovations-und Strukturökonomik (kudic@uni-bremen.de) \\ Andreas Pyka, Universität Hohenheim, Lehrstuhl für Innovationsökonomik (a.pyka@uni-hohenheim.de)
}

Dieser Artikel zeigt, wie mit Hilfe der Methode der agentenbasierten Modellierung und Simulation (ABMS) ein Beitrag zur ex-ante Policy-Beratung geleistet werden kann. Anhand eines exemplarischen Anwendungsfalls, der VISIBLE Simulationsumgebung („Virtual Simulation Lab for the Analysis of Investments in Learning and Education"), diskutieren wir die Konsequenzen unterschiedlicher Kooperationsförderinstrumente für Wissensdiffusionsprozesse in Netzwerken am Beispiel der Region Heilbronn-Franken. Die Simulationsergebnisse zeigen, dass die strukturelle Konfiguration eines regionalen Innovationssystems eine zentrale Bedeutung für die Gestaltung von Kooperationsfördermaßnahmen hat und dass Interventionen, die darauf abzielen, Wissenstransfer zwischen den Akteuren anzuregen, genau die entgegengesetzten Wirkungen entfalten können.

\section{Ex-Ante Evaluation of Investments in Knowledge, Learning, and Innovation \\ An Application Example}

Knowledge diffusion in regional innovation systems is considered as a necessary prerequisite to spur innovation and the economic performance of the actors involved. Yet, the conditions under which actors exchange knowledge in an efficient way are still not fully understood. In this paper we apply an agent-based simulation approach designed for ex-ante policy evaluation. The simulation approach and the application example are based on the VISIBLE simulation environment ("Virtual Simulation Lab for the Analysis of Investments in Learning and Education"). We investigate how presumably positive interventions affect the diffusion performance within an empirical network. Our results indicate that policy interventions can even hamper the diffusion properties of some network structures.

KEYWORDS: innovation networks, knowledge diffusion and transfer, agent-based modeling and simulation, ex-ante policy evaluation

This is an article distributed under the terms of the Creative Commons Attribution License CCBY 4.0 (https://creativecommons.org/licenses/by/4.0/)

https://doi.org/10.14512/tatup.26.3.51

Submitted: 11. 07.2017. Peer reviewed. Accepted: 18.09.2017

\section{Einleitung}

Sowohl in der Politik als auch in der Forschung herrscht heutzutage Konsens, dass Wissen, Lernen und Innovation als zentrale Erfolgsfaktoren von Unternehmen und ganzen Volkswirtschaften anzusehen sind. Umso erstaunlicher ist es, dass bis heute nur wenige gesicherte empirische Befunde existieren, die innovationspolitische Eingriffe in Wirtschaftssysteme mit defizitärem Wissenstransfer und Lernprozessen bzw. Strukturproblemen auf der Innovationssystemebene in Verbindung bringen.

Der vorliegende Beitrag veranschaulicht, wie unter Verwendung agentenbasierter Modellierung und Simulation (ABMS) eine ex-ante Evaluation von innovationspolitischen Interventionen in regionale Wirtschaftssysteme erfolgen kann. Die gewählte Herangehensweise erlaubt es, strukturelle Defizite auf systemischer Ebene aufzudecken und deutlich realistischere Anhaltspunkte für innovationspolitische Interventionen zu liefern als dies mit mikroökonomischen Standardmodellen möglich wäre.

Die nachstehenden Ausführungen basieren auf Arbeiten im Rahmen des Projektes VISIBLE (Virtual Simulation Lab for the Analysis of Investments in Learning and Education) ${ }^{1}$. Der Anstoß für die Entwicklung von VISIBLE war die Einsicht, dass politische Entscheidungsträger sich unentwegt mit komplexen und durch ein hohes Maß an Unsicherheit gekennzeichneten Entscheidungssituationen konfrontiert sehen. In der Praxis werden Entscheidungen zumeist auf Basis vergangenheitsbasierter Informationen, Erfahrungen sowie intuitiver Entscheidungsheuristiken getroffen. Wird jedoch in bestimmten Tätigkeitsfeldern Neuland betreten, so werden unterstützende Systeme erforderlich, die Informationen intelligent miteinander verbinden und die Hinweise auf die zu erwartenden komplexen Dynamiken liefern können.

1 www.visible-projekt.de 


\section{Theoretische Fundierung von Ansätzen zur ex-ante Policy-Evaluierung}

Wissen, Lernen und Innovation sind die Kernpfeiler der Neo-Schumpeterianischen Theorie (Hanusch und Pyka 2007). Die Vertreter dieser Theorierichtung sind davon überzeugt, dass Innovationen die endogenen Triebkräfte des wirtschaftlichen Wandels sind. Ökonomische Akteure handeln begrenzt rational, verfolgen individuelle Strategien des Wissensstockaufbaus - beispielsweise durch organisationale Lernprozesse, inter-organisationale Wissensaustauschprozesse im Rahmen von Kooperationen und Netzwerken - und (re-)kombinieren unter Einsatz organisationaler Routinen und individueller Fähigkeiten alte sowie neue Wissensbestandteile, sodass neues Wissen bzw. Innovationen entstehen können. Kurzum, es wird ein enger Zusammenhang zwischen individuellem Akteursverhalten, Wissen, Innovation und wirtschaftlicher Entwicklung unterstellt.

Die theoretische und empirische Forschung der letzten Jahre zeigt, dass nicht alle Unternehmen in gleichem Maße in der Lage sind, Wissen zu nutzen. Bereits Anfang der 1990er-Jahre wurde das Konzept der absorptiven Kapazitäten (absorptive capacity) vorgestellt (Cohen und Levinthal 1990). Dahinter steht die Überlegung, dass Unternehmungen über bestimmte Fähigkeiten verfügen müssen, um relevantes Wissen wahrzunehmen, zu absorbieren und schließlich auch gewinnbringend anzuwenden, so beispielsweise in Form neuartiger und vermarktungsfähiger Produkte und Dienstleitungen. Entscheidend ist allerdings, dass eine solche Absorptionsfähigkeit nicht einfach vorausgesetzt werden kann, sondern im Zeitverlauf erworben wird. Dabei sind unter anderem auch die historischen Pfade wichtig, entlang derer sich das Unternehmen entwickelt hat. Da jedes Unternehmen eine einzigartige Historie aufweist, sind auch die Lernprozesse, die diese vollzogen haben, einzigartig. Nooteboom et al. (2007) weisen zudem auf die Bedeutung der kognitiven Distanz hin. Sind die Wissensbasen der betrachteten Unternehmen zu ähnlich, so wird kein indirekt miteinander verbunden sind. Die Verbindungen dienen dem Austausch von Ideen, Informationen, Wissen und Expertise. Netzwerke sind eingebunden in eine breitere sozioökonomische Umgebung und haben eine strategische Dimension insofern, als dass die eingebundenen Akteure kooperieren, um bestehendes Wissen zu (re-)kombinieren, neues Wissen zu schaffen und schließlich neuartige Güter und Dienstleistungen zu generieren, die auf eine gewisse Marktnachfrage stoßen.

\section{ABMS als Modellierungsansatz zur Politikberatung}

Konventionelle Methoden stoßen schnell an ihre Grenzen, wenn es darum geht, die Konsequenzen von Eingriffen in komplexe adaptive Systeme aufzudecken und zu analysieren. Daher gewinnt seit geraumer Zeit die Methode der agentenbasierten Modellierung und Simulation (ABMS) zunehmend an Bedeutung in den Wirtschafts- und Sozialwissenschaften (Dawid 2006; Pyka und Fagiolo 2007; Mueller und Pyka 2017).

Übertragen auf die Analyse von Innovationsprozessen ermöglicht die Methodik der ABMS eine Herangehensweise, in der das individuelle Verhalten, Wissen der einzelnen Akteure (Haushalte, Konsumenten, Firmen, Forschungseinrichtungen, etc.) eines Wirtschaftssystems im Vordergrund stehen. Ökonomische Akteure können als Bündel von Wissensbestandteilen verstanden werden, die durch individuelle Wissensaustauschund Lernstrategien ihren Wissensstock ausbauen, um so die notwendigen Voraussetzungen für die Realisierung innovativer Produkte und Dienstleitungen zu schaffen. Mit anderen Worten: Wissen kann explizit berücksichtigt werden und Innovationen stellen keinen exogenen Effekt dar. Stattdessen sind Innovationen sowohl Ergebnis als auch Grundlage für Entscheidungen der Akteure in einem komplexen und dynamischen Innovationssystem (Wooldridge und Jennings 1995).

\section{Zu komplexe Modelle können leicht zu bedeutungslosen Konstrukten verkommen.}

Wissensaustausch stattfinden, da es kaum Komplementaritäten gibt. Sind die Wissensbasen zu unterschiedlich, wird ebenfalls kein Wissensaustausch zustande kommen, da die Akteure keinen gemeinsamen Nenner finden. Folglich herrschen lediglich in jenen Unternehmen, die sich zwischen diesen beiden Extremen bewegen, die notwendigen Voraussetzungen, um unternehmensübergreifende Wissenstransferprozesse zu initiieren.

Wir konzentrieren uns nachstehend auf Innovationsnetzwerke. Bezugnehmend auf Cantner und Graf (2011) sowie Brenner et al. (2011) definiert Kudic (2015) Innovationsnetzwerke wie folgt: Innovationsnetzwerke bestehen aus einer wohldefinierten Anzahl ökonomischer Akteure, die entweder direkt oder
Durch die stetig steigende Leistungsfähigkeit computergestützter Simulationen können immer komplexere Modelle implementiert und analysiert werden, sodass ABMS auch für politische Entscheidungssituationen zunehmend an Bedeutung gewinnen. In diesem Zusammenhang erfreut sich insbesondere die sogenannte ,ex-ante Evaluation innovationspolitischer Interventionen in regionale Innovationssysteme " eines großen Interesses. ABMS-Ansätze erlauben es, eine sehr große Anzahl an heterogenen Agenten zu modellieren, deren Verhalten auf verschiedene Weise voneinander abhängt. Wirtschaftspolitische Eingriffe können in einer möglichst realitätsnahen virtuellen Umgebung simuliert werden, bevor sie in der Realität zum Einsatz kommen. 
Die zugrundeliegende Frage nach der optimalen Komplexität eines agentenbasierten Modells ist eine der zentralen Herausforderungen im Rahmen der Modellkonzeption. Hierbei sieht sich der Anwender dem Konflikt ausgesetzt, ein Modell zu erschaffen, welches zwar einfach, aber gleichzeitig hinreichend komplex ist, um neue Erkenntnisse zu generieren. So besteht z. B. bei zu komplexen Modellen immer die Gefahr, dass wir die Modelle und Prozesse innerhalb der Modelle ebenso wenig verstehen wie diejenigen, die wir in der Realität erklären wollen (Axtell und Epstein 1994). Zu komplexe Modelle können dementsprechend leicht zu bedeutungslosen Konstrukten verkommen. Das in diesem Beitrag beschriebene Wissensdiffusionsmodell ist dementsprechend als zweckmäßige, hinreichend komplexe Abbildung (Starfield 1990) eines regionalen Wirtschaftssystems zu verstehen.

\section{Die VISIBLE Simulationsumgebung}

\section{Gegenstand und Umfang von VISIBLE}

Das Projekt VISIBLE zielt darauf ab, eine virtuelle Simulationsumgebung für die ex-ante Evaluation von innovationspolitischen Eingriffen in regionale Innovationssysteme aufzubauen. Dieser Ansatz ermöglicht es, die Wirksamkeit unterschiedlicher Investitionsalternativen in Wissen, Wissensaufbau und Bildung unter möglichst realitätsnahen Bedingungen zu untersuchen, bevor eine dieser Maßnahmen in der Realität zur Anwendung kommt.

Die VISIBLE Simulationsumgebung (Abb. 1) umfasst drei Ebenen: eine Simulationsebene, eine empirische Ebene und eine kontextuelle Ebene. Im Kern der Simulationsebene stehen verschiedene, eng aufeinander abgestimmte Simulationsmodule, die es erlauben, verschiedene Facetten eines regionalen Innovationssystems systematisch zu analysieren. Im Rahmen des Projektes VISIBLE wurden die Simulationsmodule für die Pilotregion Heilbronn-Franken exemplarische getestet und angewendet. Die Module können sowohl einzeln als auch in Kombination verwendet werden. Die konzeptionelle Basis der VISIBLE Simulationsumgebung zeichnet sich dadurch aus, dass die Akteure heterogen sind, idiosynkratrischen Wissenstransfer- und Lernstrategien folgen und im Modell durch individuelle, quantitativ und qualitativ veränderbare Wissensvektoren $\left(\right.$ kene $\left.^{2}\right)$ repräsentiert werden (Gilbert et al. 2001). Gleichzeitig fußt VISI-

2 Kenes erlauben neben inkrementellen Verbesserungen der unternehmerischen Wissensbasis und dem experimentellen Ausprobieren bislang nicht in der Wissensbasis enthaltenen Wissens den wechselseitigen und absichtsvollen Austausch von Wissenselementen in Innovationskooperationen.
BLE auf einer breit diversifizierten Datenbasis, die empirische Eckdaten und Besonderheiten der untersuchten Pilotregion Heilbronn-Franken bereitstellt und alle notwendigen Informationen liefert, um simulationsrelevante Indikatoren und Parameter zu generieren und bei Bedarf die jeweiligen Einzelsimulationsläufe mit empirischen Daten anzureichern.

In welchem Umfang Module und Daten der VISIBLE Simulationsumgebung im Rahmen von Einzelsimulationsläufen Verwendung finden, hängt von der zugrunde gelegten Fragestellung $\mathrm{ab}$, die es in enger Abstimmung mit den Stakeholdern abzuleiten gilt. Ähnlich wie in verhaltensökonomischen Realexperimenten werden auch in den von uns erzeugten ,in-silicio“-Laboren, unter kontrollierbaren Rahmenbedingungen, ceteris paribus Systemeingriffe simuliert und im Rahmen sogenannter Szenarioanalysen systematisch ausgewertet. In der Praxis hat sich herausgestellt, dass die Akzeptanz der Simulationsergebnisse maßgeblich davon abhängt, ob es im Vorfeld der Simulationsdurchläufe gelingt, die Anforderungen der Stakeholder möglichst präzise in Simulationsszenarien zu übersetzen. Demzufolge verstehen wir die Schnittstelle zu den Stakeholdern als integralen Bestandteil der VISIBLE Simulationsumgebung.

\section{Modellbeschreibung des Simulationsmodells}

Das VISIBLE Modul zur Analyse von Wissensdiffusion baut auf einem aus der Literatur bekannten Modell auf (Cowan und Jonard 2004; Mueller et al. 2017). Das ursprüngliche Modell bedient sich zur Analyse von Wissensdiffusionsprozessen eines Barter-Trade-Diffusionsprozesses ${ }^{3}$, welcher insbesondere in informellen Netzwerken eine realistische Annahme darstellt. Das

3 Im Gegensatz zur unfreiwilligen Wissensweitergabe durch technologische Spillover-Effekte wird hier zwischen den Akteuren wechselseitig Wissen ausgetauscht. 


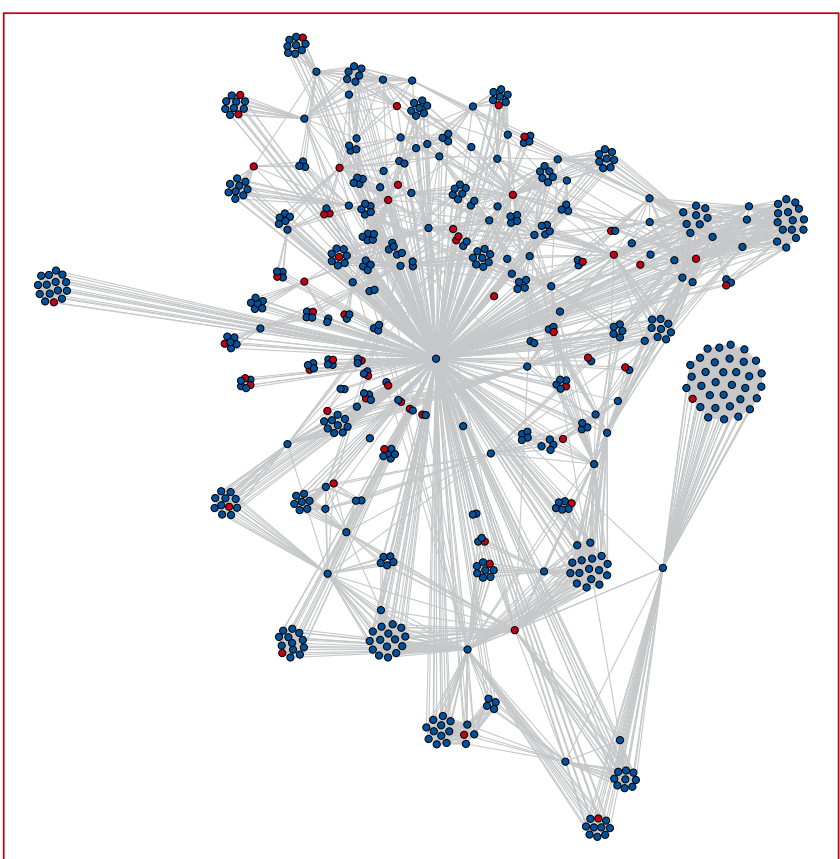

Abb.2: Möglicher Wissenszuwachs einer Firma für verschiedene $\Delta_{\max }$ Knoten in Rot: Firmen der Region; Knoten in Blau: Firmen außerhalb der Region; visualisiert mit Gephi 0.9.1. Quelle: Eigene Darstellung

Wissensdiffusionsmodul des VISIBLE Projektes dagegen zielt darauf $a b$, die Diffusionsprozesse innerhalb formeller Netzwerke zu untersuchen.

Theoretische und konzeptionelle Vorarbeiten zur Absorptionsfähigkeit von Firmen zeigen, dass die Fähigkeit zur Identifikation und Nutzbarmachung von Wissen von zentraler Bedeutung für deren Innovationserfolg ist (Cohen und Levinthal 1990). Dementsprechend zielen Akteure in unserem Modell in erster Linie darauf ab, komplementäre Wissensbestände zu erkennen, um anschließend über Wissensaustauschprozesse ihren Wissensstock zu erweitern. Während der Initialisierung der Simulation wird jede Firma $I \in\{1, \ldots, N\}$ eines Netzwerkes mit einem Wissensvektor $v_{i}=\left(v_{i, c}\right)$ bestehend aus $c \in\{1, \ldots, K\}$ Kategorien ausgestattet. Die miteinander verbundenen Firmen tauschen im Verlauf der Simulation solange ihr Wissen aus, bis keine Firma mehr von ihren Partnern lernen kann. Die Fähigkeit zweier Akteure, Wissen auszutauschen, hängt nicht nur von der individuellen Absorptionsfähigkeit ab, sondern auch von der jeweiligen kognitiven Distanz der Akteure, d. h. dem Unterschied zwischen den Wissensniveaus der Firmen. Aufbauend auf den Arbeiten von z. B. Nooteboom et al. (2007) nehmen wir dementsprechend an, dass Firmen nur voneinander lernen können (d. h. die Fähigkeit, Wissen anderer Firmen in den eigenen Wissensstock aufzunehmen und $\mathrm{zu}$ integrieren), wenn der Unterschied der Wissensniveaus einer Wissenskategorie weder zu gro $\beta$ noch $z u$ klein ist.

In jedem Simulationsschritt $t=1, \ldots, T$ tauschen die Firmen ihr Wissen mit im Netzwerk direkt verbundenen Firmen aus. Für zwei Firmen $i, j \in\{1, \ldots, N\}$ mit $i \neq j$ gilt, dass Firma $j$ in al- len Wissenskategorien von Firma $i$ Wissen bekommen könnte, in denen Firma $i$ höheres Wissen aufweist als Firma $j$ und vice versa. Dies bedeutet allerdings nicht, dass Firma $j$ das Wissen der anderen Firmen auch aufnehmen kann. Wenn der Unterschied zwischen den Wissensniveaus der Firmen $\left(\left|\left(v_{i, c}-v_{j, c}\right)\right|\right)$ größer als die maximale kognitive Distanz $\Delta_{\max }$ ist, nehmen wir an, dass Firma $j$ das Wissen von Firma $i$ nicht integrieren kann. Für den Fall, dass der Unterschied kleiner $\Delta_{\max }$ ist (wenn gilt $\left.\left|\left(v_{i, c}-v_{j, c}\right)\right| \leq \Delta_{\max }\right)$, nehmen wir weiter an, dass die Fähigkeit, neues Wissen in den eigenen Wissensstock zu integrieren, einen umgekehrt u-förmigen Verlauf annimmt (vgl. Abb. 2). Für den Wissenszuwachs in Periode $t=1$ einer Firma $j$ gilt:

$c_{j, t=1}=\left(1-\frac{\left|\left(v_{i, c, t=0}-v_{j, c, t=0}\right)\right|}{\Delta_{\max }}\right) \cdot \frac{\left|\left(v_{i, c, t=0}-v_{j, c, t=0}\right)\right|}{\Delta_{\max }}$

\section{Ausgewählte Simulationsergebnisse}

Ein Blick auf die gängige Förderpraxis auf Bundesebene (BMBF 2017) sowie auf die europäischer Ebene (EU 2017) zeigt, dass sich die finanzielle Förderung von Forschungs- und Entwicklungsverbünden über die letzten Jahrzehnte zu einem wichtigen Förderinstrument entwickelt hat. Dabei werden Vorhaben mehrerer, zu einem Verbund zusammengeschlossener Partner aus Wirtschaft und Wissenschaft für eine begrenzte Zeit gefördert, wobei bis heute weitestgehend unklar ist, welchen Einfluss die strukturellen Eigenschaften dieser Verbünde (d. h. Größe, Heterogenität der Akteure, Projektdauer etc.) auf das Gesamtsystem haben, in dem sie eingebunden sind. Auch regionalen Entscheidungsträgern steht dieses Förderinstrument zur Verfügung, um die Kooperationsintensität ausgewählter Akteure zu stimulieren. So stellt sich die Frage, wie passgenaue Kooperationsförderinstrumente zu gestalten sind.

Um die Möglichkeiten des Simulationsmodells zu verdeutlichen, analysieren wir im folgenden Experiment mögliche Konsequenzen einer politischen Intervention in ein bestehendes Kooperationsnetzwerk der Firmen in der Region Heilbronn-Franken (Abb. 3). Die verwendeten Daten stammen aus dem VISIBLE Projekt und speisen sich aus mehreren Primär- und Sekundärdatenerhebungen (BMBF 2017; EU 2017; VISIBLE 2016). Das hier betrachtete Netzwerk stellt die größten zusammenhängenden Komponenten der Firmen in der Region dar und besteht aus 558 Firmen, die über 3554 Kanten miteinander verbunden sind, wobei lediglich 58 Firmen innerhalb der Region Heilbronn-Franken lokalisiert sind. Sobald Firmen in mindestens einem EU- oder BMBF-Verbundvorhaben als Verbundpartner in Erscheinung treten, wird eine Kooperationsbeziehung unterstellt. Als Bezugsjahr wurde das Jahr 2013 gewählt.

Die folgende Szenarioanalyse zielt darauf ab, die Effekte zu analysieren, welche bei verschiedenen Interventionen zur Kooperationsförderung innerhalb eines Netzwerkes entstehen können. Die verwendeten Parametereinstellungen sind Tabelle $1 \mathrm{zu}$ entnehmen. Abbildung 4 zeigt den durchschnittlichen Wissensstand der Firmen im Modell über 300 Simulationsschritte für verschiedene Szenarien. Um mögliche Zufallseffekte zu vermei- 


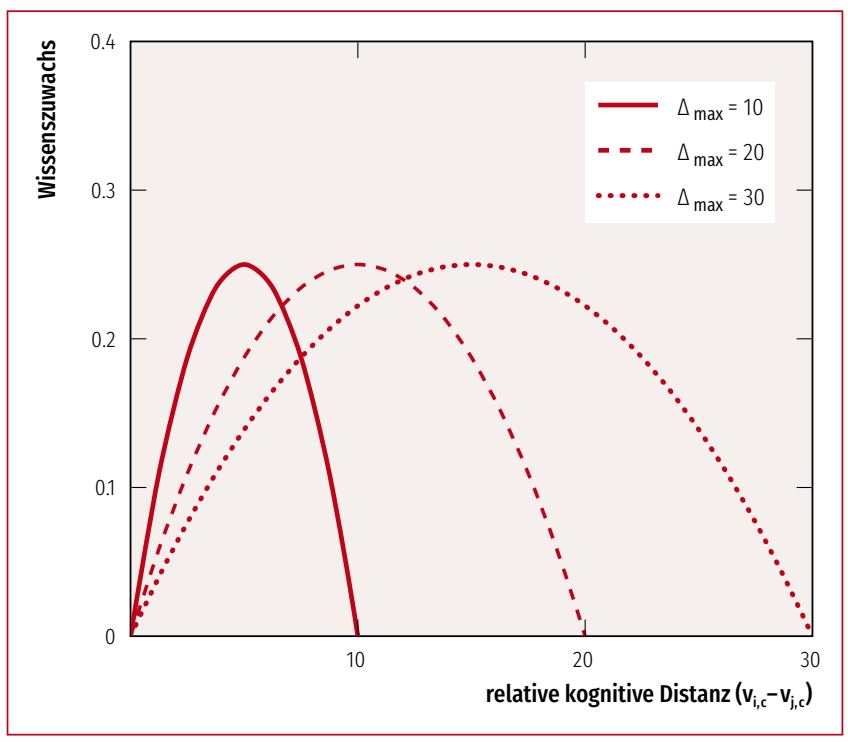

Abb.3: Empirisches Netzwerk der Region Heilbronn-Franken, 2013.

Quelle: Eigene Darstellung

den, sind die präsentierten Ergebnisse die ermittelten Durchschnittsverläufe über je 500 Simulationsläufe mit identischen Einstellungen.

Das erste Szenario „keine Intervention“ beinhaltet das empirische Netzwerk ohne eine Intervention und dient somit als Referenzszenario. Für die anderen drei Szenarien wurden während der Initialisierung der Simulation gezielt 100 neue Verknüpfungen zwischen den Firmen generiert, wobei je nach Szenario zwischen einer bestimmten Gruppe von Firmen Verbindungen eingefügt wurden.

So wurden für das Szenario „kleine Unternehmen“ zunächst die $10 \%$ mit der geringsten Anzahl an Verbindungen identifiziert und anschließend die neuen Verbindungen zwischen dieser Gruppe Firmen eingefügt. Für das Szenario ,große Unternehmen" wurden wiederum Verbindungen zwischen den $10 \%$ Firmen mit der höchsten Anzahl an bestehenden Verbindungen gewählt. Im Szenario „mittlere Unternehmen“ wurden die Firmen ausgewählt, welche weder zu den „kleinen“ noch zu den ,großen“ Firmen zählen. Kurzum, die hier eingeführte und nachstehend verwendete Größendifferenzierung stellt auf die Eingebundenheit der Akteure, gemessen anhand der Anzahl direkter Verbindungen, $a b$.

Abbildung 4 zeigt, dass zum Ende der Simulation $(t=300)$ insbesondere die auf kleine abzielende Interventionen sich als positiv gegenüber dem Referenzszenario erweist. Die Ergebnisse deuten auf einen interessanten Effekt hin. Zunächst einmal gilt festzuhalten, dass die Netzwerkstruktur einen großen Einfluss auf die Wissensdiffusionsprozesse innerhalb des Gesamtsystems hat. Obwohl bei allen drei Interventionen die gleiche Anzahl an Verbindungen neu geschaffen wurde, zeigen die Ergebnisse, dass ein erheblicher Unterschied zwischen den jeweiligen Eingriffen existiert. Des Weiteren ist ersichtlich, dass sich im Zeitablauf die Ergebnisse verschieben und dass es sogar

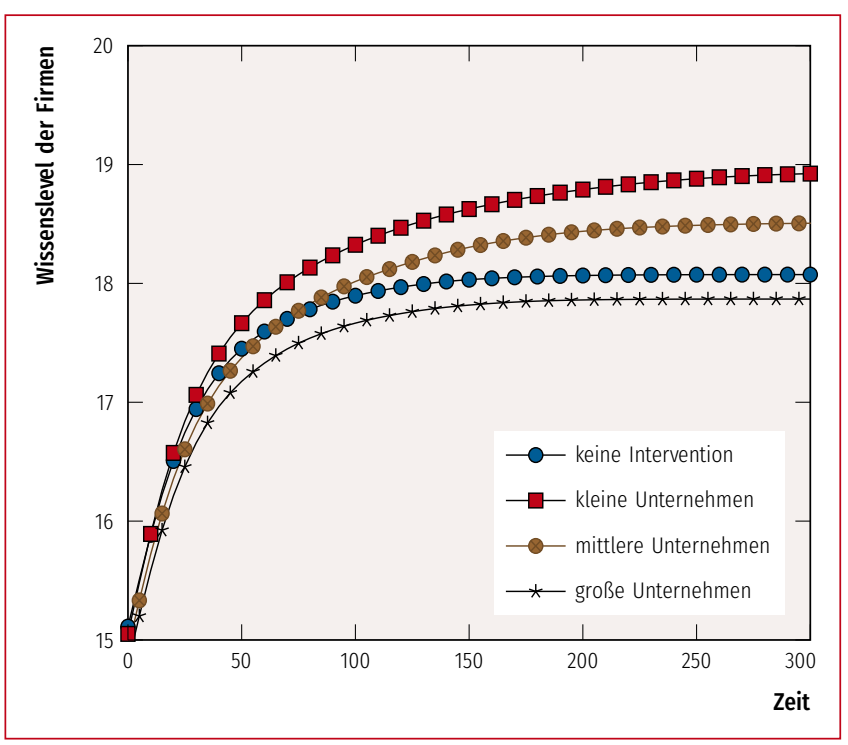

Abb. 4: Wissenslevel der Firmen für verschiedene Interventionen.

Quelle: Eigene Darstellung

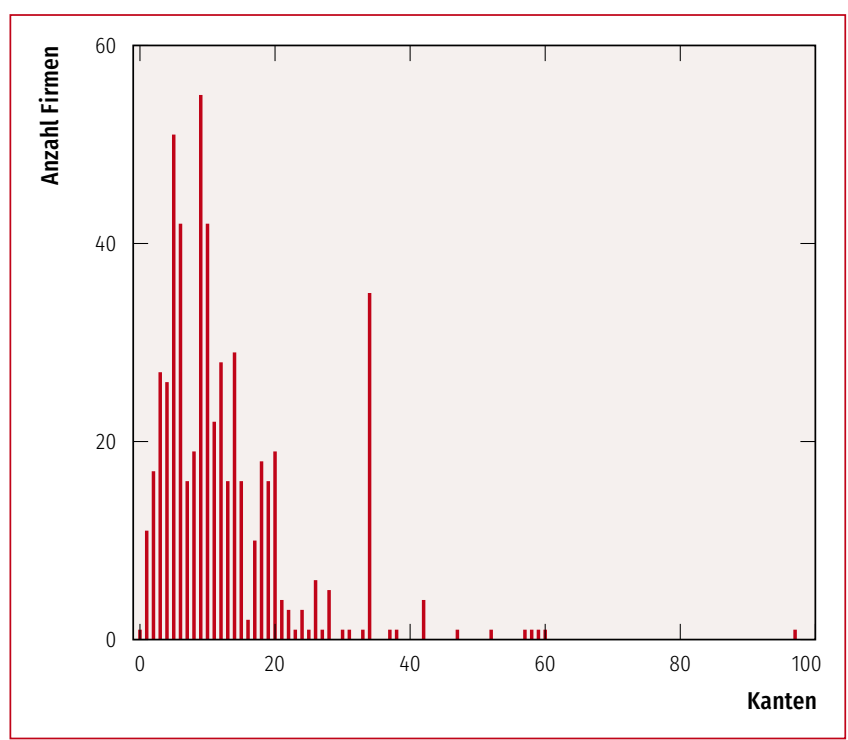

Abb.5: Kantenverteilung des empirischen Netzwerkes.

Quelle: Eigene Darstellung

Parameterbeschreibung Ausprägung

\begin{tabular}{|l|l|}
\hline Größe des Wissensvektors c & 10 \\
\hline $\begin{array}{l}\text { Wissenslevel zu Beginn } \\
\text { der Simulation }\end{array}$ & Gleichverteilt $\{0<x<30\}$ \\
\hline Maximale kognitive Distanz $\Delta_{\max }$ & 7 \\
\hline
\end{tabular}

Tab.1: Parametereinstellungen der Simulation

Quelle: Eigene Darstellung 
Interventionen gibt, die dem eigentlichen Ziel des Eingriffs entgegenstehen und die Diffusionseigenschaften des Systems negativ beeinflussen können.

Die Begründung für dieses Ergebnis liegt in einer Netzwerkeigenschaft, deren Bedeutung in der Literatur oft vernachlässigt wird: die sogenannte degree distribution des empirischen Netzwerkes. In Abbildung 5 ist die Verteilung der Kanten auf die Firmen im Netzwerk dargestellt.

Abbildung 5 zeigt uns, dass sehr wenige Firmen über sehr viele Verbindungen verfügen und gleichzeitig sehr viele Firmen eine geringe Anzahl an Verbindungen haben. Gut verbundene Firmen können allerdings sehr schnell einen hohen Wissensstock aufbauen, während weniger gut verbundene Firmen verwendet werden können. Ihre Stärke liegt vielmehr, wie im Anwendungsbeispiel demonstriert, in der Identifikation unerwarteter Effekte und Systemdynamiken, die mit einfachen linearen Modellen nicht zu erkennen sind.

Zusätzliche Erklärungspotenziale von ABMS sind durch eine zielgerichtete Erhebung, Aufbereitung und Nutzung empirischen Daten zu erwarten. Neben der explorativen Datenverwendung, die in erster Linie dazu dient, strukturelle Besonderheiten einer Region zu quantifizieren, sehen wir in der instrumentellen Nutzung von Daten einen besonderen Mehrwert. Derzeit werden empirische Daten in erster Linie dazu genutzt, die Startbedingungen agentenbasierter Simulationen möglichst realitätsnah zu initialisieren. Inwiefern es in den kommenden Jahren zu einer

\section{Um Wissensdiffusionsprozesse in Netzwerken zu fördern, ist eine differenzierte Förderstrategie notwendig.}

im Wettbewerb um neues Wissen zurückbleiben. Dieser Unterschied führt dazu, dass die Firmen im Netzwerk nicht mehr voneinander lernen und der Diffusionsprozess abbricht.

Interventionen wie die oben genannten beeinflussen ebendiese Eigenschaft des Netzwerkes und können somit den Unterschied zwischen gut verbundenen Firmen und weniger verbundenen Firmen verstärken (wie im Szenario „große Firmen“), aber auch verkleinern. Das Simulationsmodell zeigt uns also einen interessanten Effekt, welcher bei der Gestaltung wirtschaftspolitischer Eingriffe berücksichtigt werden muss.

\section{Fazit und Ausblick}

Das Ziel des vorliegenden Beitrags ist es, eine mögliche Anwendung neuer simulationsbasierter Unterstützungssysteme für die Praxis der wirtschaftspolitischen Entscheidungsfindung aufzuzeigen. Das verwendete Diffusionsmodell zeigt, dass bereits recht einfache und relativ abstrakte Modelle einen Beitrag zu einer ex-ante Evaluation von innovationspolitischen Interventionen in regionale Wirtschaftssysteme leisten können. Das verwendete Modell stellt ohne Frage eine starke Vereinfachung der Realität dar. Dennoch konnte mit diesem Ansatz und unter Verwendung ausgewählter empirischer Daten gezeigt werden, dass eine differenzierte Förderstrategie notwendig ist, um Wissensdiffusionsprozesse in Netzwerken zu fördern. Mit anderen Worten, ein One-fits-all-Förderansatz, der regionalspezifische Systemeigenschaften vernachlässigt, kann unter bestimmten Umständen sogar zu negativen Ergebnissen führen. Ein für sich genommen bemerkenswertes Ergebnis, das bis heute in der wirtschaftspolitischen Praxis kaum Berücksichtigung findet.

Gleichzeitig muss betont werden, dass ABMS nicht zur punktgenauen Vorhersage und exakten Quantifizierung wirtschaftspolitischer Interventionen in komplexe adaptive Systeme weiteren Integration der Daten- und Simulationsebene kommen wird, ist zum jetzigen Zeitpunkt noch nicht vollständig absehbar. Klar ist jedoch, dass die aktuell beobachtbaren Forschungserfolge in den Bereichen Big Data und Textmining bereits in naher Zukunft interessante Möglichkeiten der ex-ante Policy-Beratung eröffnen werden.

\section{Danksagung}

Wir bedanken uns bei der Dieter Schwarz Stiftung für die finanzielle Förderung des Projektes VISIBLE. Zudem danken wir den Gutachtern für konstruktive und hilfreiche Kommentare.

\section{Literatur}

Axtell, Robert L.; Epstein, Joshua M. (1994): Agent-Based Modeling: Understanding Our Creations. In: The Bulletin of the Santa Fe Institute 9 (2), S.28-32. Online verfügbar unter http://samoa.santafe.edu/media/bulletin_pdf/ Winter1994Bulletin.pdf, zuletzt geprüft am 24.10.2017.

Brenner, Thomas; Cantner, Uwe; Graf, Holger (2011): Innovation Networks: Measurement, Performance and Regional Dimensions. In: Industry \& Innovation 18 (1), S.1-5.

Cantner, Uwe; Graf, Holger (2011): Innovation Networks: Formation, Performance and Dynamics. In: Cristiano Antonelli (Hg.): Handbook on the Economic Complexity of Technological Change. Celtenham: Edward Elgar Publishing, S. 366-394.

Cohen, Wesley M.; Levinthal, Daniel A. (1990): Absorptive Capacity: A New Perspective on Learning and Innovation. In: Administrative Science Quarterly, 35 (1), S. 128-152.

Cowan, Robin; Jonard, Nicolas (2004): Network Structure and the Diffusion of Knowledge. In: Journal of Economic Dynamics and Control 28 (8), S. 1557-1575.

Dawid, Herbert (2006): Agent-Based Models of Innovation and Technological Change. In: Leigh Tesfatsion und Kenneth Judd (Hg.): Handbook of Computational Economics II: Agent-Based Computational Economics. Amsterdam: North-Holland, S. 1235-1272. 
Gilbert, Nigel; Pyka, Andreas; Ahrweiler, Petra (2001): Innovation Networks: A Simulation Approach. In: Journal of Artificial Societies and Social Simulation 4 (3), S. 1-14. Online verfügbar unter http://jasss.soc.surrey. ac.uk/18/4/5.html, zuletzt geprüft am 24.10.2017.

Hanusch, Horst; Pyka, Andreas (2007): Elgar Companion to Neo-Schumpeterian Economics. Cheltenham: Edward Elgar Publishing.

Kudic, Muhamed (2015): Innovation Networks in the German Laser Industry: Evolutionary Change, Strategic Positioning, and Firm Innovativeness. Heidelberg: Springer.

Mueller, Matthias; Bogner, Kristina; Buchmann, Tobias; Kudic, Muhamed (2017): The Effect of Structural Disparities on Knowledge Diffusion in Networks: An Agent-Based Simulation Model. In: Journal of Economic Interaction and Coordination 12 (3), S. 613-634.

Mueller, Matthias; Pyka, Andreas (2017): Economic Behaviour and Agent-Based Modelling. In: Roger Frantz, Shu-Heng Chen, Kurt Dopfer, Floris Heukelom und Shabnam Mousavi (Hg.): Routledge Handbook of Behavioral Economics. Abingdon: Routledge, S. 405-415.

Nooteboom, Bart; van Haverbeke, Wim; Duysters, Geert; Gilsing, Victor; van den Oord, Ad (2007): Optimal Cognitive Distance and Absorptive Capacity. In: Research Policy 36 (7), S. 1016-1034.

Pyka, Andreas; Fagiolo, Giorgio (2007): Agent-Based Modelling: A Methodology for Neo-Schumpeterian Economics. In: Horst Hanusch und Andreas Pyka (Hg.): The Elgar Companion to Neo-Schumpeterian Economics. Cheltenham: Edward Elgar Publishing, S. 467-492.

Starfield, Anthony M. (1990): Qualitative, Rule-Based Modeling. In: Bioscience 40 (8), S. 601-604.

Wooldridge, Michael; Jennings, Nicholas R. (1995): Intelligent Agents: Theory and Practice. In: The Knowledge Engineering Review 10 (2), S. 115-152.

\section{Forschungsdaten}

BMBF - Bundesministerium für Bildung und Forschung (2017): Förderkatalog. Online verfügbar unter http://www.foerderportal.bund.de/foekat, zuletzt geprüft am 01.06.2017.
EU - Europäische Kommission (2017): CORDIS. Forschungs- \& Entwicklungsinformationsdienst der Gemeinschaft. Online verfügbar unter http://www. cordis.europa.eu, zuletzt geprüft am 01.06.2017.

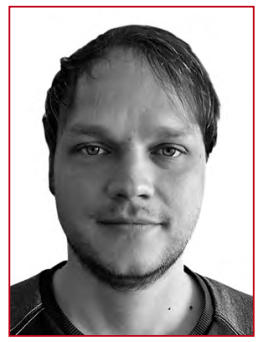

\section{DR. MATTHIAS MÜLLER}

arbeitet seit 2010 als wissenschaftlicher Mitarbeiter an der Universität Hohenheim. Zuvor studierte er Wirtschaftswissenschaft an der Universität Bremen. 2016 schloss er erfolgreich seine Promotion an der Universität Hohenheim ab.

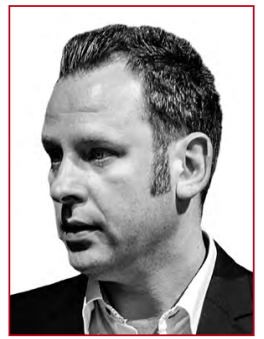

\section{DR. MUHAMED KUDIC}

promovierte 2012 an der Universität Hohenheim. Von 2013 bis 2016 war er wissenschaftlicher Referent und Projektleiter beim Stifterverband für die deutsche Wissenschaft. Seit 2017 bekleidet er eine Postdocstelle an der Universität Bremen.

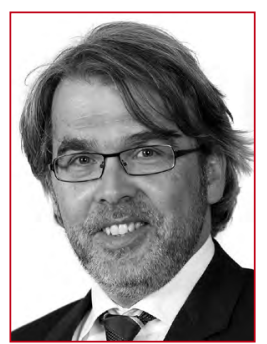

\section{PROF. DR. ANDREAS PYKA}

arbeitet seit Sommer 2009 am Fachgebiet für Innovationsökonomik der Universität Hohenheim. Nach der Promotion an der Universität Augsburg verbrachte er zwei Jahre als Postdoc am Institut Nationale de la Recherche Agronomique in Grenoble, habilitierte sich 2004 und erhielt 2006 einen Ruf an die Universität Bremen.
Nachhaltigkeit

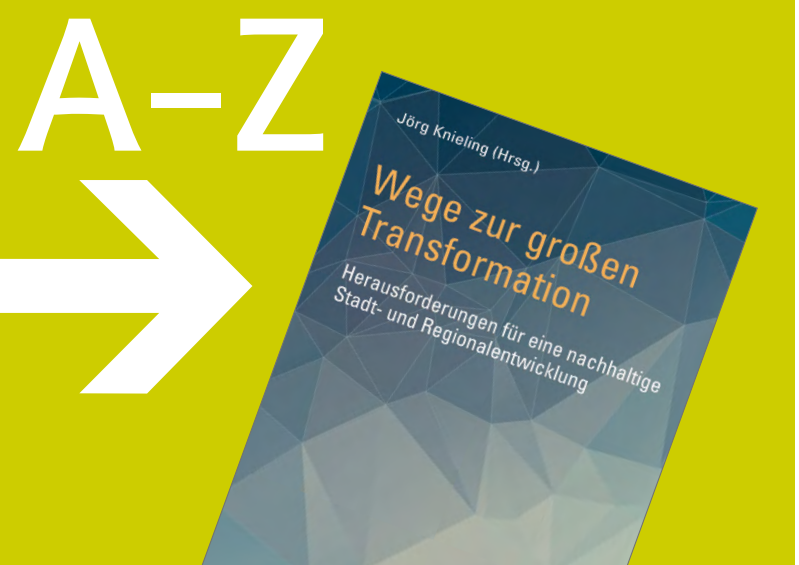

\section{wie Handlungsmöglichkeiten}

In Zeiten von Klimawandel, Digitalisierung und Urbanisierung stehen Städte und Regionen weltweit vor großen Herausforderungen. Wie kann vor diesem Hintergrund eine nachhaltige Stadt- und Regionalentwicklung aussehen? Welche Chancen und Potenziale bieten Städte und Regionen für die geforderte "große Transformation"? Junge Wissenschaftlerinnen und Wissenschaftler aus verschiedenen Disziplinen beleuchten mögliche Wege und Ansatzpunkte.

\section{J. Knieling}

\section{Wege zur großen Transformation}

Herausforderungen für eine nachhaltige Stadt- und Regionalentwicklung 240 Seiten, broschiert, mit zahlreichen Abbildungen, 24,95 Euro, ISBN 978-3-96006-026-0

Erhältlich im Buchhandel oder versandkostenfrei innerhalb Deutschlands bestellbar unter www.oekom.de

Die guten Seiten der Zukunft II OeKOM 